\title{
Deleterious processes of a diode-pumped cesium vapor hollow-core photonic-crystal fiber laser
}

\author{
Guofei An, You Wang, Juhong Han, He Cai, Zhigang Jiang, Ming Gao, Shunyan Wang, Wei Zhang, \\ Hongyuan Wang, Liangping Xue, and Jie Zhou \\ Southwest Institute of Technical Physics, Chengdu, Sichuan 610041, China \\ (Received 29 April 2016; revised 21 June 2016; accepted 9 September 2016)
}

\begin{abstract}
A diode-pumped alkali laser (DPAL) provides the significant promise for high-powered performances. In this paper, a mathematical model is introduced for examination of the kinetic processes of a diode-pumped cesium vapor hollow-core photonic-crystal fiber (HC-PCF) laser, in which the cesium vapor is filled in the center hole of a photonic-bandgap fiber instead of a glass cell. The influence of deleterious processes including energy pooling, photo-ionization, and Penning ionization on the physical features of a fiber DPAL is studied in this report. It has been theoretically demonstrated that the deleterious processes cannot be ignored in a high-powered fiber-DPAL system.
\end{abstract}

Keywords: DPAL; hollow core; ionization; photonic bandgap

\section{Introduction}

In the recent years, a diode-pumped alkali laser (DPAL) has been extensively studied due to the potential for its excellent physical features ${ }^{[1-3]}$. The oscillator of a DPAL usually contains an enclosed glass cell, in which the vapor of neutral alkali ( $\mathrm{K}, \mathrm{Rb}, \mathrm{Cs}$ ) and several kinds of buffer gases (helium and hydrocarbon with small molecular weight) are sealed with a certain pressure ${ }^{[4-6]}$. In 2006, Payne et al. proposed a patent describing a new kind of DPAL in which a hollow-core photonic-crystal fiber (HC-PCF) is used as the vapor container ${ }^{[7]}$. Unlike a normal DPAL, such an integrated design is thought to have higher stability. And the optical coupling efficiency of this system can be improved significantly comparing with the traditional configuration. Slepkov et al. also demonstrated the possibility of introducing alkali vapor into a standard hollow-core fiber with sufficient gas density ${ }^{[8]}$. Recently, Sintov et al. calculated the physical features of a rubidium vapor-based fiber laser using a simple mathematical model, in which the HC-PCFDPAL was treated as a three-level laser system ${ }^{[9]}$.

Generally, alkalis are the most easily ionized atomic species, especially for rubidium and cesium. Figure 1 illustrates the energy level diagram for Cs atoms. The electrons in cesium atoms are pumped from the $6^{2} S_{1 / 2}$ level to the $6^{2} P_{3 / 2}$ level corresponding to the $D_{2}$ line

Correspondence to: Y. Wang, Southwest Institute of Technical Physics, Renmin South Road 4-7, Chengdu, Sichuan 610041, China. Email: youwang_2007@aliyun.com

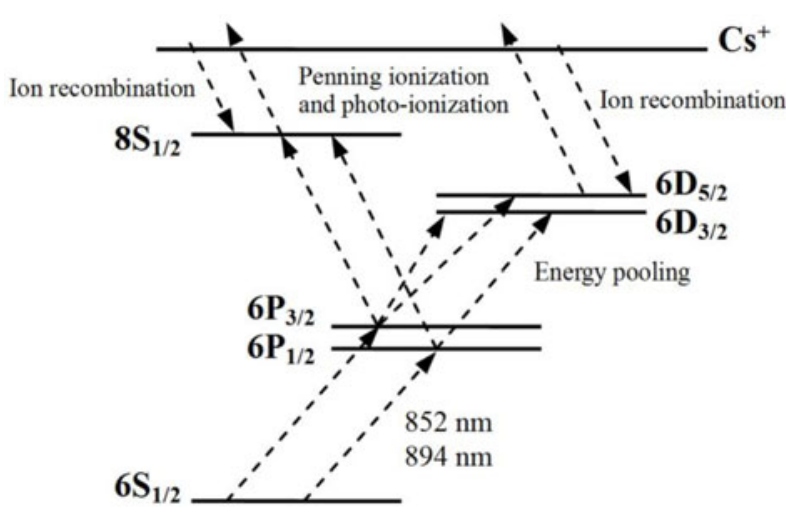

Figure 1. Energy level diagram for a cesium atom.

$(852.3 \mathrm{~nm})$. After a rapid relaxation in the fine structure with the aid of some alkanes with small hydrocarbon molecules, electrons fall back to the ground state with stimulated emission corresponding to the $D_{1}$ line $(894.6 \mathrm{~nm})$. Under the condition of high-powered pump, the electrons will be excited to the higher levels $\left(6^{2} D_{5 / 2,3 / 2}\right.$ and $\left.8^{2} S_{1 / 2}\right)$ by energy pooling collisions. Further, ionization processes including photo-ionization and Penning ionization will occur on the $6^{2} D_{5 / 2,3 / 2}$ and $8^{2} S_{1 / 2}$ levels resulting in decrease of the density of neutral atoms.

To deeply study the kinetics of an HC-PCF-DPAL, we construct a quasi-five-level model with the cesium vapor as a gain medium in this report. The deleterious processes of energy pooling and ionization are taken into account in 


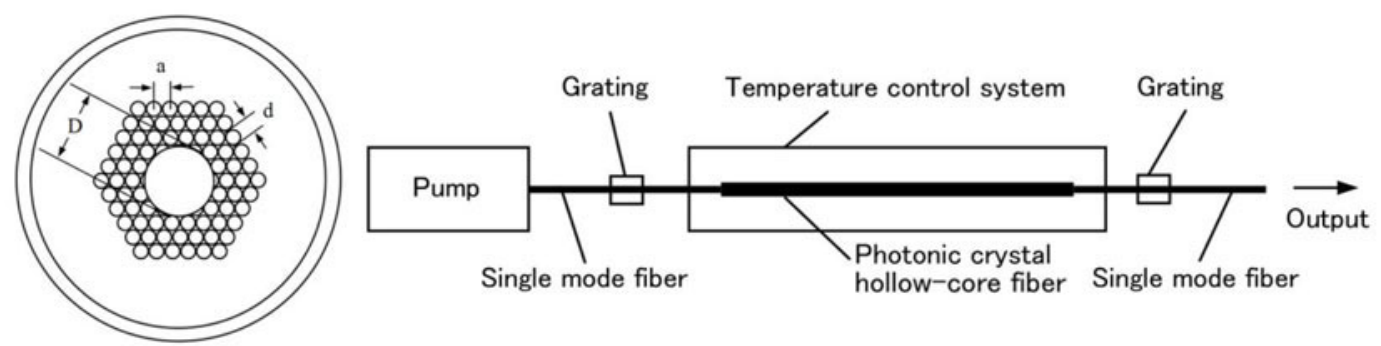

(a)

(b)

Figure 2. (a) Transverse view of an HC-PCF and (b) the schematic diagram of the diode-pumped cesium vapor HC-PCF laser system.

the theoretical model. The absorbed power, output power, and optical-to-optical efficiency versus the pump power have been evaluated. In addition, the influences of energy pooling, Penning ionization and Photo-ionization on the output features of a DPAL are also evaluated. To the best of our knowledge, there have not been any similar reports about a diode-pumped cesium vapor HC-PCF laser.

\section{Theoretical analyses}

Figure 2(a) shows the transverse view of an HC-PCF, which consists of a center hollow core with the diameter $D$ and several rings circular hole (honeycomb lattice) with the diameter $d$ and the hole-to-hole spacing $a$. By properly choosing the geometric parameter values, a wide bandgap guiding can be realized ${ }^{[10]}$. The schematic diagram of this laser system is shown in Figure 2(b). Cesium vapor and buffer gases are introduced into the center hole of an HC-PCF, which is connected with two single mode fibers (SMFs).

By taking the processes of energy pooling, photoionization, Penning ionization, and recombination into account, the three-level rate equations used in our previous simulation are extended to quasi-five-level rate equations. We designate the number densities of the $6^{2} S_{1 / 2}, 6^{2} P_{1 / 2}$, $6^{2} P_{3 / 2}, 6^{2} D_{5 / 2,3 / 2}$ and $8^{2} S_{1 / 2}$, and ionized levels to be $n_{1}, n_{2}, n_{3}, n_{4}$ and $n_{5}$, respectively. Therefore, the total number density $n_{0}$ can be expressed as $n_{0}=n_{1}+n_{2}+n_{3}+$ $n_{4}+n_{5}$. The appropriate rate equations can be expressed as follows $^{[11]}$ :

$$
\begin{aligned}
\frac{\mathrm{d} n_{1}}{\mathrm{~d} t}= & -\Gamma_{P}+\Gamma_{L}+\frac{n_{2}}{\tau_{D_{1}}}+\frac{n_{3}}{\tau_{D_{2}}}+\frac{n_{4}}{\tau_{4}} \\
& +k_{E P 2}\left(n_{2}\right)^{2}+k_{E P 3}\left(n_{3}\right)^{2} \\
& +k_{P I} n_{4}\left(n_{2}+n_{3}\right), \\
\frac{\mathrm{d} n_{2}}{\mathrm{~d} t}= & -\Gamma_{L}+\gamma_{32}\left(T_{w}\right)\left\{\left[n_{3}-n_{2}\right]\right. \\
& \left.-\left[2 \exp \left(-\frac{\Delta E}{k_{B} T_{w}}\right)-1\right] n_{2}\right\}-\frac{n_{2}}{\tau_{D_{1}}} \\
& -2 k_{E P 2}\left(n_{2}\right)^{2}-k_{P I} n_{2} n_{4},
\end{aligned}
$$

$$
\begin{aligned}
\frac{\mathrm{d} n_{3}}{\mathrm{~d} t}= & \Gamma_{P}-\gamma_{32}\left(T_{w}\right)\left\{\left[n_{3}-n_{2}\right]\right. \\
& \left.-\left[2 \exp \left(-\frac{\Delta E}{k_{B} T_{w}}\right)-1\right] n_{2}\right\}-\frac{n_{3}}{\tau_{D_{2}}} \\
& -2 k_{E P 3}\left(n_{3}\right)^{2}-k_{P I} n_{3} n_{4} \\
\frac{\mathrm{d} n_{4}}{\mathrm{~d} t}= & k_{E P 2} \cdot\left(n_{2}\right)^{2}+k_{E P 3} \cdot\left(n_{3}\right)^{2}-\frac{n_{4}}{\tau_{4}} \\
& -k_{P I} n_{4}\left(n_{2}+n_{3}\right)-\Gamma_{\text {photo-ionization }} \\
& +k_{\text {recombination }}\left(n_{5}\right)^{3} \\
\frac{\mathrm{d} n_{5}}{\mathrm{~d} t}= & k_{P I} n_{4}\left(n_{2}+n_{3}\right)+\Gamma_{\text {photo-ionization }} \\
& -k_{\text {recombination }}\left(n_{5}\right)^{3}, \\
\Gamma_{\text {photo-ionization }}= & n_{4} \sigma_{\text {photo-ionization }}\left(\frac{I_{l}}{h v_{l}}+\frac{I_{P}}{h v_{p}}\right)
\end{aligned}
$$

where $\Gamma_{P}$ is the stimulated absorption transition rate caused by pump photons, $\Gamma_{L}$ is the transition rate of laser emission, $\Gamma_{\text {photo-ionization }}$ is the transition rate of photo-ionization, $\gamma_{32}\left(T_{w}\right)$ is the fine-structure relaxation rate, $\Delta E$ is the energy gap between the ${ }^{2} P_{3 / 2}$ and ${ }^{2} P_{1 / 2}$ levels with the value of $554 \mathrm{~cm}^{-1}, k_{B}$ is the Boltzmann constant, $\tau_{D 1}$ is the $D_{1}\left(6^{2} P_{1 / 2} \rightarrow 6^{2} S_{1 / 2}\right)$ radiative lifetime, $\tau_{D 2}$ is the $D_{2}$ $\left(6^{2} S_{1 / 2} \rightarrow 6^{2} P_{3 / 2}\right)$ radiative lifetime, $\sigma_{\text {photo-ionization is the }}$ photo-ionization transverse section, $k_{E P 2}\left(n_{2}\right)^{2}$ denotes the transition rate of electrons from the $6^{2} P_{1 / 2}$ to the $6^{2} D_{5 / 2,3 / 2}$ and $8^{2} S_{1 / 2}$ levels, $k_{E P 3}\left(n_{3}\right)^{2}$ denotes the transition rate from the $6^{2} P_{3 / 2}$ level to the $6^{2} D_{5 / 2,3 / 2}$ and $8^{2} S_{1 / 2}$ levels, $k_{P I}$ is the Penning ionization rate coefficients, $k_{\text {recombination }}$ is the recombination rate constant, $I_{P}$ and $I_{l}$ are the pump and laser intensities given by Ref. [12] and $\tau_{4}$ is the lifetime of $6^{2} D_{5 / 2,3 / 2}$ and $8^{2} S_{1 / 2}$ levels ${ }^{[13]}$, respectively. Note that we assume the lifetimes of $6^{2} D_{5 / 2,3 / 2}$ and $8^{2} S_{1 / 2}$ levels are of the same value to simplify the calculation. A summary of critical parameters of this model are listed in Table 1. Note that the transmission loss of the hollow-core fiber is set to 0.82 in the theoretical calculation.

\section{Results and discussions}

To systematically investigate the influence of deleterious processes on the physical features of a DPAL, we list five 
Table 1. Summary of critical parameters in our model.

\begin{tabular}{lll}
\hline Parameter description & Value & Reference \\
\hline Partial pressure of $\mathrm{He}$ & 478.8 Torr & This work \\
Partial pressure of $\mathrm{C}_{2} \mathrm{H}_{6}$ & 100 Torr & This work \\
Cell length $L$ & $2 \mathrm{~m}$ & This work \\
Cell radius $R$ (hollow core of an HC-PCF) & $50 \mu \mathrm{m}$ & This work \\
Transverse section of the $D_{2}$ line $\sigma_{D_{2}}$ & $1.53 \times 10^{-9} \mathrm{~cm}^{2}$ & {$[13]$} \\
Transverse section of the $D_{1}$ line $\sigma_{D_{1}}$ & $2.31 \times 10^{-9} \mathrm{~cm}^{2}$ & {$[13]$} \\
Photo-ionization cross section $\sigma_{\text {photo-ionization }}$ & $2.00 \times 10^{-17} \mathrm{~cm}^{2}$ & {$[14]$} \\
$D_{1}$ radiative lifetime $\tau_{D_{1}}$ & $34.9 \mathrm{~ns}$ & {$[13]$} \\
$D_{2}$ radiative lifetime $\tau_{D_{2}}$ & $30.5 \mathrm{~ns}$ & {$[13]$} \\
Lifetime of $6^{2} D_{5 / 2,3 / 2}$ and $8^{2} S_{1 / 2}$ levels $\tau_{4}$ & $60 \mathrm{~ns}$ & {$[14]$} \\
Energy pooling rate constant $k_{E P 2}$ & $7.1 \times 10^{-10} \mathrm{~cm}^{3} \mathrm{~s}^{-1}$ & {$[15]$} \\
Energy pooling rate constant $k_{E P 3}$ & $2.8 \times 10^{-10} \mathrm{~cm}^{3} \mathrm{~s}^{-1}$ & {$[15]$} \\
Penning ionization rate constant $k_{P I}$ & $1.0 \times 10^{-8} \mathrm{~cm}^{3} \mathrm{~s}^{-1}$ & {$[16]$} \\
Recombination rate constant $k_{\text {recombination }}$ & $7.124 \times 10^{-21} \mathrm{~cm}^{6} \mathrm{~s}^{-1}$ & {$[17]$} \\
\hline
\end{tabular}

Table 2. Different cases investigated in the model.

\begin{tabular}{lccc}
\hline No. & Energy pooling & Penning ionization & Photo-ionization \\
\hline Case 1 & $\times$ & $\times$ & $\times$ \\
Case 2 & $\sqrt{ }$ & $\times$ & $\times$ \\
Case 3 & $\sqrt{ }$ & $\sqrt{ }$ & $\times$ \\
Case 4 & $\sqrt{ }$ & $\times$ & $\sqrt{ }$ \\
Case 5 & $\sqrt{ }$ & $\sqrt{ }$ & $\sqrt{ }$ \\
\hline
\end{tabular}

cases in Table 2. Case 1 denotes a simple three-level system investigated in the absence of excitation to the upper states $6^{2} D_{5 / 2,3 / 2}$ and $8^{2} S_{1 / 2}$ and their ionization. Case 2 represents the state with considering the process of energy pooling. Case 3 describes the processes of energy pooling and Penning ionization. Case 4 illustrates the occurrence when energy pooling and photo-ionization are considered. Case 5 reflects the circumstance including all of these three processes.

In Figure 3, the laser power is given as a function of the pump power. It can be seen that all the quantities of the curves monotonically increase with the pump level when the pump power is less than $50 \mathrm{~W}$. When the pump power exceeds $50 \mathrm{~W}$, the output powers of Cases 1-5 reach a plateau since the pump absorption already achieves the saturated status in an HC-PCF cell. In addition, different curves in Figure 3 show the contribution of different processes to the output power. Comparing the curve of Case 1 with that of Case 2, there is almost no difference between them, which indicates that the process of energy pooling has little influence on the output power. For the curves of Cases 3 and 4 , the output decreases are $1.92 \%$ and $13.90 \%$ with the pump power of $100 \mathrm{~W}$, respectively. The reason of this is the decrease of the number density of neutral alkali atoms caused by the process of ionization. Further, when considering both processes of Penning ionization and photo-ionization in the model (Case 5), the curve decreases by $13.92 \%$ with the pump power of $100 \mathrm{~W}$. This result suggests that photoionization provide greater influence than Penning ionization.

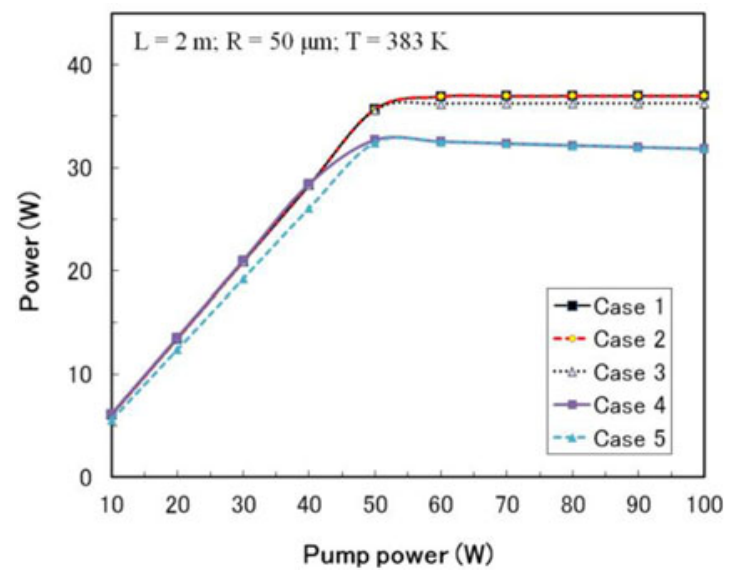

Figure 3. Output power versus pump power in different cases.

Figure 4 shows the absorbed power, output power, and optical-to-optical efficiency as functions of the pump power when the temperature is set to $110^{\circ} \mathrm{C}$. As shown in Figure 4, the maximal optical-to-optical efficiency can be obtained at pump power of $50 \mathrm{~W}$ in both Cases 1 and 5 . In the range of 50-100 $\mathrm{W}$ for pump power, the optical-to-optical efficiency decreases with increase of the pump power due to the saturation of output power. Further, the maximal optical-to-optical efficiency of Case $5(64.87 \%)$ is lower than that of Case $1(71.72 \%)$ because of the effect of deleterious processes.

Unlike other types of lasers, the density of a gas-state gain medium inside the hollow fiber is extremely sensitive to the ambient temperature ${ }^{[18]}$. In Figure 5, the absorbed power, output power, and population density are given as functions of the fiber temperature. The fiber length and pump power are set to $2 \mathrm{~m}$ and $100 \mathrm{~W}$, respectively. It can be seen that the population density increases with the temperature in Figure 5. However, saturation occurs at the temperature higher than $120^{\circ} \mathrm{C}$ for the curves corresponding to both the absorbed power and the output power. It 


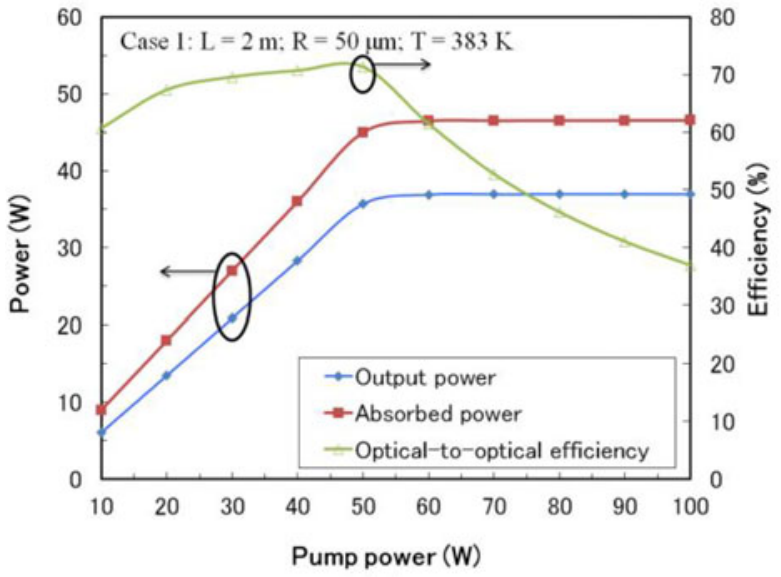

(a)

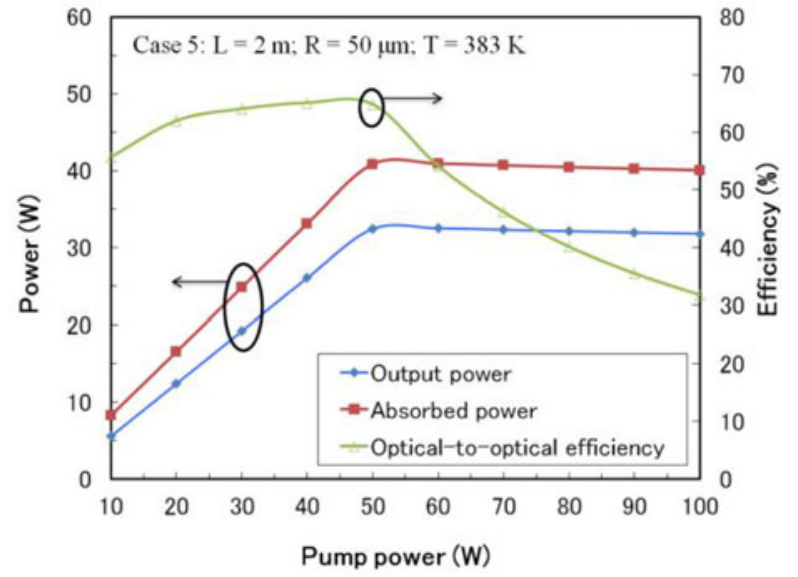

(b)

Figure 4. Absorbed power, output power, and optical-to-optical efficiency versus pump power in (a) Case 1 and (b) Case 5.

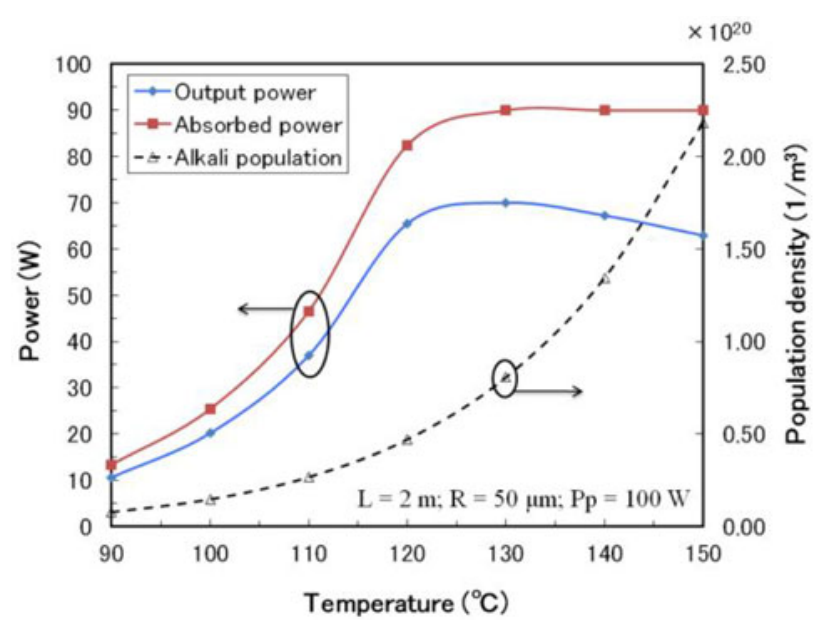

Figure 5. Absorbed power, output power, and population density $\left(n_{0}\right)$ versus temperature of Case 1 .

means that the absorbed power or the output power cannot be greatly improved when the temperature is higher than $120^{\circ} \mathrm{C}$ although the total population density is exponentially increased inside the hollow fiber. To further increase the output power above $120^{\circ} \mathrm{C}$, the pump power should be enhanced.

\section{Summary}

In this study, we develop a mathematical model to calculate the static-state DPALs using an HC-PCF as the vapor cell. The effect of deleterious processes including energy pooling, Penning ionization, and photo-ionization on the output features of a DPAL are theoretically investigated. We also study how the temperature influences the output power and alkali population. The results reveal that the output power will decrease because of the deleterious processes, and such a phenomenon becomes serious with the even higher pump power. It has also been found that the density of the alkali vapor inside the fiber cell is extremely sensitive to the ambient temperature. In other words, the temperature is a critical factor in this new kind of laser system.

\section{References}

1. Y. Wang, T. Kasamatsu, Y. Zheng, H. Miyajima, H. Fukuoka, S. Matsuoka, M. Niigaki, H. Kubomura, T. Hiruma, and H. Kan, Appl. Phys. Lett. 88, 141112 (2006).

2. W. F. Krupke, Prog. Quantum Electron. 36, 4 (2012).

3. B. V. Zhdanov and R. J. Knize, Opt. Eng. 52, 021010 (2013).

4. W. F. Krupke, R. J. Beach, V. K. Kanz, and S. A. Payne, Opt. Lett. 28, 2336 (2003).

5. B. D. Barmashenko and S. Rosenwaks, J. Opt. Soc. Am. B 30, 1118 (2013).

6. W. Zhang, Y. Wang, H. Cai, L. P. Xue, J. H. Han, H. Y. Wang, and Z. Y. Liao, Appl. Opt. 53, 4180 (2014).

7. S. A. Payne, R. J. Beach, J. Dawson, and W. F. Krupke, U.S. patent (July 25, 2006).

8. A. D. Slepkov, A. R. Bhagwat, V. Venkataraman, P. Londero, and A. L. Gaeta, Opt. Express 16, 18976 (2008).

9. Y. Sintov, D. Malka, and Z. Zalevsky, Opt. Lett. 39, 4655 (2014).

10. Y. Y. Wang, N. V. Wheeler, F. Couny, P. J. Roberts, and F. Benabid, Opt. Lett. 36, 669 (2011).

11. G. F. An, Y. Wang, J. H. Han, H. Cai, J. Zhou, W. Zhang, L. P. Xue, H. Y. Wang, M. Gao, and Z. G. Jiang, Opt. Express 23, 26414 (2015).

12. D. A. Steck, Cesium D Line Data, available: http://steck.us/al kalidata.

13. R. J. Knize, B. V. Zhdanov, and M. K. Shaffer, Opt. Express 19, 7894 (2011).

14. Z. J. Jabbour, R. K. Namiotka, J. Huennekens, M. Allegrini, S. Milosevic, and F. de Tomasi, Phys. Rev. A 54, 1372 (1996).

15. J. Huennekens, Z. Wu, and T. Walker, Phys. Rev. A 31, 196 (1985).

16. S. S. Q. Wu, Hydrocarbon-Free Resonance Transition $795 \mathrm{~nm}$ Rubidium Laser (University of California, San Diego, 2009).

17. G. P. Perram and G. D. Hager, 42nd AIAA Plasmadynamics and Lasers Conference, Honolulu, Hawaii, 27-30 June (2011), paper 2011-4002.

18. C. V. Sulham, G. P. Perram, M. P. Wilkinson, and D. A. Hostutler, Opt. Commun. 283, 4328 (2010). 